\title{
What is expanded in progressive myoclonus epilepsy?
}

$\mathrm{T}^{\mathrm{t}}$ has been discovered that the gene Ifor cystatin B (CSTB) on chromosome $21 \mathrm{q} 22.3$ is responsible for progressive myoclonus epilepsy of Unverricht-Lundborg type (EPM1; ref. 1). Comprehensive mutation analyses failed to identify most of the mutations in unrelated patients $s^{2,3}$, and it was suggested that a common mutation might be an unstable sequence in the $5^{\prime}$ region of the gene ${ }^{3}$. Recently, it was shown that the common mutation mechanism in patients from various geographic and ethnic origins appears to be an expansion of a repeated DNA sequence in the $5^{\prime}$ flanking region of $C S T B^{4,5}$. However, the exact nature of the repeated sequence is controversial; it is either an expansion of novel 18- and 15-mer minisatellites ${ }^{4}$ or an expansion of a polymorphic dodecamer repeat CCCCGCCCCGCG ${ }^{5}$. The resolution of the nature of the repeat has important implications for the mechanism of this expansion in EPM1 (ref. 6).

If the expanded sequence consisted of the reported 15- and 18-mers, restriction enzymes SacI and DdeI should cleave the expanded DNA. Thus, we have digested two patientderived CSTB genomic clones containing the larger mutant EPM1 alleles. This experiment clearly shows that the proposed minisatellite sequences reported by Virtaneva and colleagues ${ }^{4}$ are not present in our clones (Fig. 1), as these two enzymes failed

$\begin{array}{cccc}\text { 18-mer } & T & T \text { T A T T } \\ \text { 12-mer } & \text { CxCCxCGCCxCxCxGxCX } \\ \text { 15-mer } & T & \text { T } & \text { A A A }\end{array}$

Fig. 2 A comparison of the dodecamer repeat expanded in EPM1 with the microsatellite repeats reported by Virtaneva et $a l^{4}$. An ' $x$ ' has been inserted into the sequence of the dodecamer to allow for the alignment of the sequences. The additional bases in the reported 18 -mer and 15 mer, which result from cross-banding, are shown above and below, respectively.

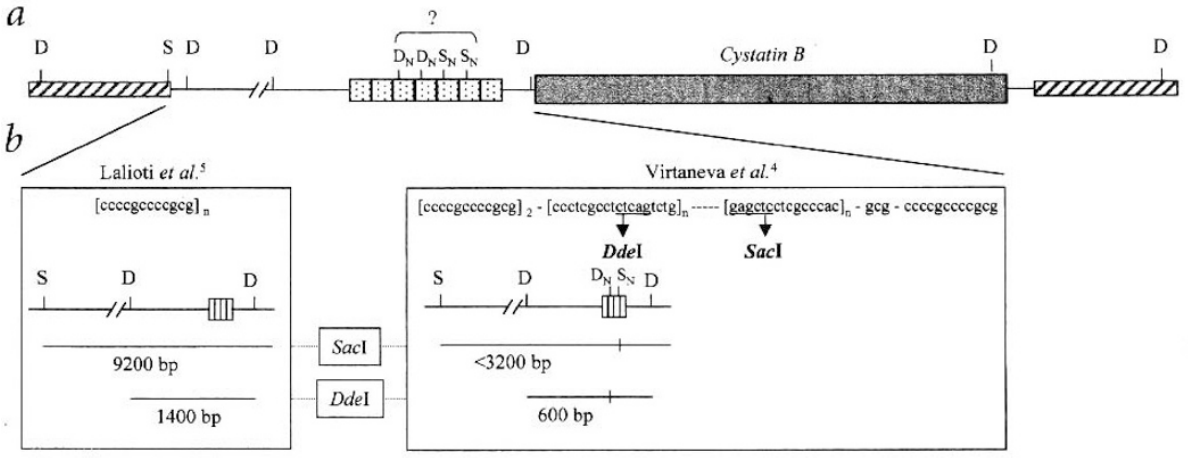

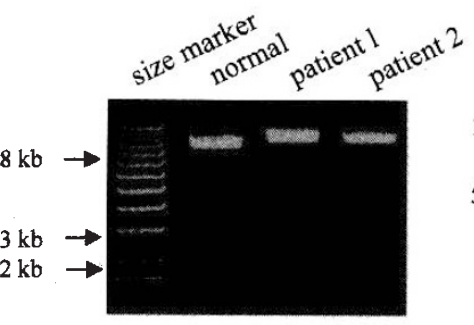

SacI

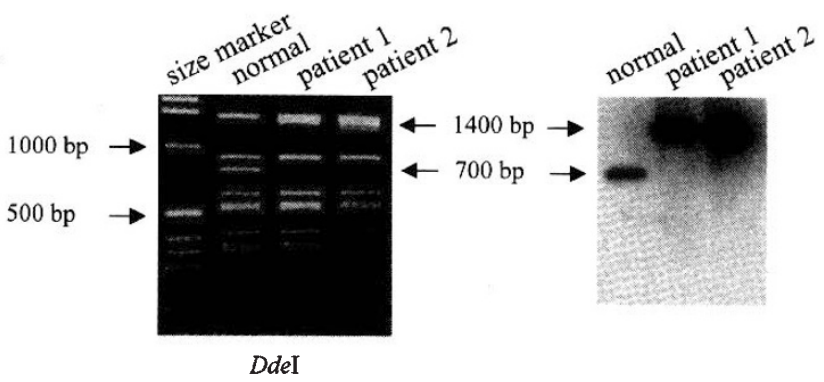

DdeI

Fig. 1 a A schematic representation of the clones containing the expanded CSTB alleles (not to scale). The vectorderived sequences are cross-hatched, CSTB is grey, and the repeats are represented as boxes. Sacl (S) and Ddel (D) novo 15- and 18-mer minisatellite sequences of Virtaneva et al. ${ }^{4} \boldsymbol{b}$, Expected fragments produced by digestion of wo different mutant clones, both contain expanded repeats. If the 15-mer were present, it would introduce multiple Sacl sites into the insert-producing two fragments, one smaller than $3.2 \mathrm{~kb}$ and the other approximately $6 \mathrm{~kb}$. If only the dodecamer were present, Sacl would cut only once in the vector linearizing the plasmid as shown in c. If the 18-mer were present, Ddel digestion would produce a diagnostic fragment of 600 bp in addition to framents from the insert and vector; if it were not present, a larger band of 1,400 bp would be expected as detected in $\mathrm{c}$. Sacl and Ddel digestion of normal and mutant clones. Note the absence of the 3.2-kb (for SaCl) and digestion, Southern blotting and hybridization with an oligonucleotide containing two dodecamer repeats verified that the 700-bp normal and 1,400-bp mutant bands (indicated by the arrows) contained the repeats.

to cleave the 'expanded' DNA. The conclu- of Medical Genetics, Cantonal Hospital of sions of Virtaneva et al. may have been Geneva, 1 rue Michel-Servet, $\mathrm{CH}-1211$ Geneva 4 , drawn from the sequencing gels presented in their article (and possibly others), and we propose that they have interpreted crossbands as real nucleotides (Fig. 2). mechanism in EPM1 is the expansion of Swiss FNRS, the Swiss OFES and the University the dodecamer repeat, not the expansion and Cantonal Hospital of Geneva. M.D.L. is a of de novo 15- or 18-mer minisatelites. This trainee of the Molecular and Cellular Biology implies that the EPM1 expansion alleles graduate program of the University of Geneva were probably a result of replication errors, Martin fellowship from the NH \& MRC of as hypothesized for the trinucleotide repeat Australia. disorders ${ }^{6}$, and not produced by recombination, as for minisatellites ${ }^{7}$.

Maria D. Lalioti' ${ }^{1}$, Hamish S. Scott ${ }^{1}$, \& Stylianos E. Antonarakis ${ }^{1,2}$ ${ }^{1}$ Laboratory of Human Molecular Genetics, Department of Genetics and Microbiology, University of Geneva Medical School, ${ }^{2}$ Division

1. Pennacchio, L.A. et al. Science 271, 1731-1734 (1996). 2. Lalioti, M.D. et al. Am. J. Hum. Genet. 60, 342-351 (1997).

3. Lafrenière, R.G. et al. Nature Genet. 15, 298-302 (1997)

4. Virtaneva, K. etal. Nature Genet. 15, 393-396 (1997).

4. Virtaneva, K. et al. Nature Genet. 15, 393-396 (199

6. Mandel, J.-L. Nature 386, 767-769 (1997).

7. Buard, J. \& Jeffreys, A.J. Nature Genet. 15, 327-328 (1997). 